\title{
A New Approach for the Stability Analysis of Wave Networks
}

\author{
Ya Xuan Zhang ${ }^{1}$ and Gen Qi Xü \\ ${ }^{1}$ College of Science, Civil Aviation University of China, Tianjin 300300, China \\ ${ }^{2}$ Department of Mathematics, Tianjin University, Tianjin 300072, China
}

Correspondence should be addressed to Ya Xuan Zhang; bunnyxuan@tju.edu.cn

Received 12 February 2014; Accepted 19 April 2014; Published 11 May 2014

Academic Editor: Marco Donatelli

Copyright ( 2014 Y. X. Zhang and G. Q. Xu. This is an open access article distributed under the Creative Commons Attribution License, which permits unrestricted use, distribution, and reproduction in any medium, provided the original work is properly cited.

\begin{abstract}
We introduce a new approach to investigate the stability of controlled tree-shaped wave networks and subtrees of complex wave networks. It is motivated by regarding the network as branching out from a single edge. We present the recursive relations of the Laplacian transforms of adjacent edges of the system in its branching order, which form the characteristic equation. In the stability analysis, we estimate the infimums of the recursive expressions in the inverse order based on the spectral analysis. It is a feasible way to check whether the system is exponentially stable under any control strategy or parameter choice. As an application we design the control law and study the stability of a 12-edge tree-shaped wave network.
\end{abstract}

\section{Introduction}

The stability analysis of networks of flexible elements, such as vibrating strings, beams, membranes, and plates has been intensively and extensively studied in the past decades (see [1-3] for some general works and [4-17] for wave networks). A wave network can be asymptotically stabilized by some appropriate feedback control strategy. However, its exponential stability is difficult to check. The exponential stability of a dynamic system is usually analyzed by multiplier method $([18,19]$, etc.), resolvent estimation ([20,21], etc.), or spectral analysis and Riesz basis approach $([4,8-12,14]$, etc.). For wave networks, an appropriate multiplier has not been found so far and the resolvent is too complicated to estimate; thus the spectral analysis becomes a possible technique. In fact, suppose that the spectral determined growth assumption (SDGA) holds for a network, which means that the energy decay rate of the network is exactly the supremum of the real parts of its spectra. Then a dissipative system is exponentially stable if and only if the imaginary axis is not an asymptote of its spectra. That is, the characteristic equation $D(\lambda)$ of the network satisfies

$$
\inf _{\sigma \in \mathbb{R}}|D(i \sigma)|>0
$$

Generally speaking, most controlled wave networks satisfy SDGA under certain conditions. However, as for the spectrum of these networks, it is only known that the spectra lie in a vertical strip in the complex plane. More precise properties, such as the asymptote, of the spectra remain unknown in general. Besides, the characteristic equation has been derived for wave networks of some special types (e.g., [2] presented nice results about the characteristic equation of generic trees), but its expression form does not seem very convenient for checking (1). These are challenging problems in both stability analysis of wave networks and spectral theory of nonadjoint operators.

In this paper, we will introduce a relatively easy approach to study the exponential stability of a controlled wave network. It is valid for tree-shaped networks and subtrees of complex networks. The basic idea is motivated by the natural growth of a tree. Regarding a tree-shaped network as branching out from a single edge, we can obtain a simple form of its characteristic equation by recurrence, which leads to an easy verification of (1). Our idea is illustrated below in detail.

First we consider a tree of only one edge, the dynamic behavior of which is governed by the following wave equation 


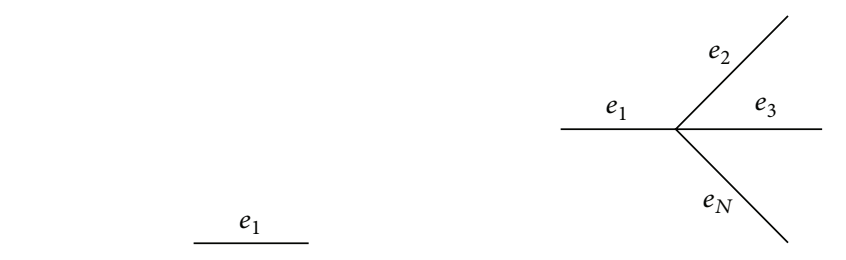

Branching out from the single-edge tree (2) Tree-shaped wave network (3)

(a)

(b)

FIgURE 1: Tree-shaped wave network (3) branching out from the single-edge tree (2).

and boundary conditions:

$$
\begin{gathered}
T_{1} y_{1, x x}(x, t)=m_{1} y_{1, t t}(x, t), \quad x \in(0,1), t>0 \\
y_{1}(0, t)=0 \\
T_{1} y_{1, x}(1, t)=R_{1}(t),
\end{gathered}
$$

where $R_{1}(t)$ denotes the extra force acting at the boundary end of the edge.

Then we consider another tree of $N$ edges, whose dynamic behavior is governed by

$$
\begin{gathered}
T_{k} y_{k, x x}(x, t)=m_{k} y_{k, t t}(x, t), \quad x \in(0,1), \\
t>0, \quad k=1,2, \ldots, N \\
y_{1}(0, t)=0 \\
y_{1}(1, t)=y_{k}(0, t), \quad k=2, \ldots, N \\
T_{1} y_{1, x}(1, t)=\sum_{k=2}^{N} T_{k} y_{k, x}(0, t) \\
T_{k} y_{k, x}(1, t)=R_{k}(t), \quad k=2, \ldots, N,
\end{gathered}
$$

where $R_{k}(t), k=2, \ldots, N$ are the extra forces acting at the corresponding boundary ends.

Comparing (2) and (3), we can regard the tree-shaped wave network (3) as branching out from the single-edge tree (2), based on the "branching force principle":

$$
R_{1}(t)=\sum_{k=2}^{N} T_{k} y_{k, x}(0, t)
$$

We can see this "branching" process clearly in Figure 1.

According to this principle, we can describe more complicated tree-shaped wave networks. Moreover, we can deduce from it the characteristic equation of the networks, which has a simple form to check (1). This can be done by the Laplacian transform of (3):

$$
\begin{gathered}
T_{k} f_{k, x x}(x, \lambda)=m_{k} \lambda^{2} f_{k}(x, \lambda), \\
x \in(0,1), \quad k=1,2, \ldots, N \\
f_{1}(0, \lambda)=0 \\
f_{1}(1, \lambda)=f_{k}(0, \lambda), \quad k=2, \ldots, N \\
T_{1} f_{1, x}(1, \lambda)=\widehat{R}_{1}(\lambda) \\
\widehat{R}_{1}(\lambda)=\sum_{k=2}^{N} T_{k} f_{k, x}(0, \lambda) \\
T_{k} f_{k, x}(1, \lambda)=\widehat{R}_{k}(\lambda), \quad k=2, \ldots, N,
\end{gathered}
$$

where $f_{k}(x, \lambda)$ and $\widehat{R}_{k}(\lambda)$ are the transforms of $y_{k}(x, t)$ and $R_{k}(t)$, respectively.

Note that for $\lambda \neq 0$, the solutions $f_{k}(x, \lambda), k=1,2, \ldots, N$ to the differential equations in (5) have the general form

$$
\begin{array}{r}
f_{k}(x, \lambda)=c_{k}\left[F_{k}(\lambda) \cosh \lambda \rho_{k} x-G_{k}(\lambda) \sinh \lambda \rho_{k} x\right], \\
\rho_{k}=\sqrt{\frac{m_{k}}{T_{k}}}, \quad k=1,2, \ldots, N,
\end{array}
$$

where $F_{k}(\lambda), G_{k}(\lambda) \in \mathbb{C}$ are arbitrary constants related to $\lambda$, and $c_{k}$ are nonzero auxiliary constants.

Substituting (6) into the first boundary condition in (5), we obtain that

$$
F_{1}(\lambda)=0
$$

Substituting (6) into $f_{k}(1, \lambda)$ and the last boundary condition in (5), we have that

$$
\begin{array}{r}
f_{k}(1, \lambda)=c_{k}\left[F_{k}(\lambda) \cosh \lambda \rho_{k}-G_{k}(\lambda) \sinh \lambda \rho_{k}\right], \\
\widehat{R}_{k}(\lambda)=\lambda T_{k} \rho_{k} c_{k}\left[F_{k}(\lambda) \sinh \lambda \rho_{k}-G_{k}(\lambda) \cosh \lambda \rho_{k}\right], \\
k=2, \ldots, N .
\end{array}
$$


From the above we can express the coefficients $F_{k}(\lambda)$ and $G_{k}(\lambda)$ by $f_{k}(1, \lambda)$ and $\widehat{R}_{k}(\lambda)$ for $k=2, \ldots, N$ as follows:

$$
\begin{array}{r}
F_{k}(\lambda) \\
=\frac{1}{c_{k} T_{k} \rho_{k}}\left[T_{k} \rho_{k} f_{k}(1, \lambda) \cosh \lambda \rho_{k}-\frac{1}{\lambda} \widehat{R}_{k}(\lambda) \sinh \lambda \rho_{k}\right], \\
G_{k}(\lambda) \\
=\frac{1}{c_{k} T_{k} \rho_{k}}\left[T_{k} \rho_{k} f_{k}(1, \lambda) \sinh \lambda \rho_{k}-\frac{1}{\lambda} \widehat{R}_{k}(\lambda) \cosh \lambda \rho_{k}\right], \\
k=2, \ldots, N,
\end{array}
$$

where $\widehat{R}_{k}(\lambda), k=2, \ldots, N$ are to be determined.

Substituting (6) into other connective conditions in (5), we get that

$$
\begin{aligned}
\widehat{R}_{1}(\lambda) & =\lambda c_{1} T_{1} \rho_{1}\left[F_{1}(\lambda) \sinh \lambda \rho_{1}-G_{1}(\lambda) \cosh \lambda \rho_{1}\right] \\
& =-\lambda \sum_{k=2}^{N} c_{k} T_{k} \rho_{k} G_{k}(\lambda) \\
f_{1}(1, \lambda) & =c_{1}\left[F_{1}(\lambda) \sinh \lambda \rho_{1}-G_{1}(\lambda) \cosh \lambda \rho_{1}\right] \\
& =c_{k} F_{k}(\lambda), \quad k=2, \ldots, N .
\end{aligned}
$$

Solving the above equations, we can express $F_{1}(\lambda)$ and $G_{1}(\lambda)$ by $F_{k}(\lambda), G_{k}(\lambda), k=2, \ldots, N$ with appropriately chosen constants $c_{k}, k=2, \ldots, N$. It remains to be determined the expressions of $F_{k}(\lambda), G_{k}(\lambda), k=2, \ldots, N$, that is, the expressions of $\widehat{R}_{k}(\lambda)$ (or equivalently $R_{k}(t)$ ), $k=2, \ldots, N$. This can be done in the following two cases.

Case 1. The network under consideration is described as (3).

Let $R_{k}(t), k=2, \ldots, N$ be the feedback controls, such as the collocated velocity feedback control $R_{k}(t)=-\alpha y_{k, x}(1, t)$, $k=2, \ldots, N$. Then we can directly get the expressions of $F_{k}(\lambda), G_{k}(\lambda), k=2, \ldots, N$ by $(9)$.

Case 2. The network under consideration is a bigger tree branching out from (3).

We proceed similarly to the boundary of the system by recurrence.

In both cases, we can finally obtain the recursive expressions of $F_{1}(\lambda)$, and hence $F_{1}(\lambda)=0$ is the characteristic equation of the system. The process is in accordance with the natural growth of a tree. In the stability analysis, we only need to estimate the infimums of each recursive expression in the inverse order to assert whether (1) holds or not. Thus the exponential stability analysis can be carried out in an easy manner. This can be similarly carried out for subtrees of complex wave networks.

The content is arranged as follows. In Section 2, we state our main results for general tree-shaped wave network and present a complete proof. In Section 3, we investigate a 12edge tree-shaped wave network as an application to the main results. In Section 4, we give a conclusion.

\section{Main Results}

In this section, we will give a rigorous statement of the main results in general. For this aim, we deal with a general subtree $\mathscr{A}$ of a complex wave network $G$. We adopt the notations introduced in [1, pp. 104] to describe a tree or a subtree.

Let $\mathscr{A}$ be a subtree (or a tree). By the degree of a vertex of $\mathscr{A}$ we mean the number of the edges that branch out from it. A vertex is called a boundary vertex if its degree is one and an interior vertex otherwise. Let $N$ be the number of the edges of $\mathscr{A}$.

Choose a boundary vertex as the root of $\mathscr{A}$, denoted by $\mathscr{R}$. The other edges and vertices are denoted by $e_{\bar{\alpha}}$ and $v_{\bar{\alpha}}$, respectively, where $\bar{\alpha}=\left(\alpha_{1}, \ldots, \alpha_{k}\right)$ is a multi-index (possibly empty) defined by recurrence in the following way. For the edge containing $\mathscr{R}$ we choose the empty index; that is, it is denoted by $e$ and its vertex different from $\mathscr{R}$ is denoted by $v$. Assume that the interior vertex $v_{\bar{\alpha}}$, contained in the edge $e_{\bar{\alpha}}$, has multiplicity $m_{\bar{\alpha}}+1$. Then there are $m_{\bar{\alpha}}$ edges, different from $e_{\bar{\alpha}}$, branching out from $v_{\bar{\alpha}}$. Denote them by $e_{\bar{\alpha} \circ \beta}$, where $\beta=1,2, \ldots, m_{\bar{\alpha}}, \bar{\alpha} \circ \beta=\left(\alpha_{1}, \ldots, \alpha_{k}, \beta\right)$, and the other vertex of the edge $e_{\bar{\alpha} \circ \beta}$ is denoted by $v_{\bar{\alpha} \circ \beta}$.

Let $\mathscr{A}_{M}$ and $\mathscr{A}_{S}$ be the set of all the interior and boundary vertices, respectively, $\mathscr{R}$ being excepted. Set $\mathscr{J}_{M}=\left\{\bar{\alpha} \mid v_{\bar{\alpha}} \epsilon\right.$ $\left.\mathscr{A}_{M}\right\}, \mathscr{J}_{S}=\left\{\bar{\alpha} \mid v_{\bar{\alpha}} \in \mathscr{A}_{S}\right\}$, and $\mathscr{J}=\mathscr{J}_{M} \cup \mathscr{J}_{S}$.

Suppose without loss of generality that the length of the edge $e_{\bar{\alpha}}$ is 1 . Then $e_{\bar{\alpha}}$ can be parameterized by its arc length by means of the function $\pi_{\bar{\alpha}}$ defined as $\pi_{\bar{\alpha}}:[0,1] \rightarrow e_{\bar{\alpha}}, \bar{\alpha} \in \mathscr{J}$ such that $\pi_{\bar{\alpha}}(0)=v_{\bar{\alpha}}$ and $\pi_{\bar{\alpha}}(1)$ is the other vertex of $e_{\bar{\alpha}}$. In this way, $e_{\bar{\alpha}}$ and its end points can be identified with the interval $[0,1]$.

Assume that a tree-shaped subnetwork coincides with $\mathscr{A}$ at rest and performs a small perpendicular vibration. Let $y^{\bar{\alpha}}=y^{\bar{\alpha}}(x, t):[0,1] \times \mathbb{R}^{+} \rightarrow \mathbb{R}$ describe the transversal displacement of $e_{\bar{\alpha}}$ at position $\pi_{\bar{\alpha}}(x)$ and time $t$. Suppose that all the interior vertices satisfy the geometric continuity condition and Kirchhoff law. Then the dynamical behavior of the subtree $\mathscr{A}$ is governed by

$$
\begin{gathered}
T^{\bar{\alpha}} y_{x x}^{\bar{\alpha}}(x, t)=m^{\bar{\alpha}} y_{t t}^{\bar{\alpha}}(x, t), \quad x \in(0,1), t>0, \bar{\alpha} \in \mathscr{J}, \\
y^{\bar{\alpha}}(1, t)=y^{\bar{\alpha} \circ \beta}(0, t), \quad \bar{\alpha} \in \mathscr{J}_{M}, \quad \beta=1,2, \ldots, m_{\bar{\alpha}}, \\
T^{\bar{\alpha}} y_{x}^{\bar{\alpha}}(1, t)=R^{\bar{\alpha}}(t), \quad \bar{\alpha} \in \mathscr{J}_{M}, \\
R^{\bar{\alpha}}(t)=\sum_{\beta=1}^{m_{\bar{\alpha}}} T^{\bar{\alpha} \circ \beta} y_{x}^{\bar{\alpha}^{\circ} \beta}(0, t), \quad \bar{\alpha} \in \mathscr{J}_{M} .
\end{gathered}
$$

The Laplacian transform of the subtree (11) is

$$
\begin{gathered}
T^{\bar{\alpha}} f_{x x}^{\bar{\alpha}}(x, \lambda)=m^{\bar{\alpha}} \lambda^{2} f^{\bar{\alpha}}(x, \lambda), \quad x \in(0,1), \bar{\alpha} \in \mathcal{J}, \\
f^{\bar{\alpha}}(1, \lambda)=f^{\bar{\alpha} \circ \beta}(0, \lambda), \quad \alpha \in \mathscr{J}_{M}, \quad \beta=1,2, \ldots, m_{\bar{\alpha}}, \\
T^{\bar{\alpha}} f_{x}^{\bar{\alpha}}(1, \lambda)=\widehat{R}^{\bar{\alpha}}(\lambda), \quad \bar{\alpha} \in \mathscr{J}_{M}, \\
\widehat{R}^{\bar{\alpha}}(\lambda)=\sum_{\beta=1}^{m_{\bar{\alpha}}} T^{\bar{\alpha} \circ \beta} f_{x}^{\bar{\alpha}^{\circ} \beta}(0, \lambda), \quad \bar{\alpha} \in \mathscr{J}_{M},
\end{gathered}
$$


where $f^{\bar{\alpha}}(x, \lambda)$ and $\widehat{R}^{\bar{\alpha}}(\lambda)$ are the transforms of $y^{\bar{\alpha}}(x, t)$ and $R^{\bar{\alpha}}(t)$, respectively.

According to the differential equations in (12), we can set

$$
\begin{array}{r}
f^{\bar{\alpha}}(x, \lambda)=c^{\bar{\alpha}}\left[F^{\bar{\alpha}}(\lambda) \cosh \lambda \rho^{\bar{\alpha}} x-G^{\bar{\alpha}}(\lambda) \sinh \lambda \rho^{\bar{\alpha}} x\right], \\
\rho^{\bar{\alpha}}=\sqrt{\frac{m^{\bar{\alpha}}}{T^{\bar{\alpha}}}}, \quad \bar{\alpha} \in \mathscr{J},
\end{array}
$$

where the coefficients $c^{\bar{\alpha}}$ are nonzero auxiliary coefficients; $F^{\bar{\alpha}}(\lambda), G^{\bar{\alpha}}(\lambda)$ are arbitrary constants related to $\lambda$, which are to be determined as recursive expressions.

For any fixed $\bar{\alpha} \in \mathscr{J}_{M}$, we consider the edge $e^{\bar{\alpha}}$ and the $m_{\bar{\alpha}}$ edges $e^{\bar{\alpha} \diamond \beta}, \beta=1,2, \ldots, m_{\bar{\alpha}}$, which branch out from $e^{\bar{\alpha}}$. Their vibration is governed by

$$
\begin{gathered}
T^{\bar{\alpha}} y_{x x}^{\bar{\alpha}}(x, t)=m^{\bar{\alpha}} y_{t t}^{\bar{\alpha}}(x, t), \quad x \in(0,1), \quad t>0 \\
T^{\bar{\alpha} \circ \beta} y_{x x}^{\bar{\alpha} \circ \beta}(x, t)=m^{\bar{\alpha} \beta} y_{t t}^{\bar{\alpha} \circ \beta}(x, t), \quad x \in(0,1), \\
t>0, \quad \beta=1,2, \ldots, m_{\bar{\alpha}} \\
y^{\bar{\alpha}}(1, t)=y^{\bar{\alpha} \circ \beta}(0, t), \quad \beta=1,2, \ldots, m_{\bar{\alpha}} \\
T^{\bar{\alpha}} y_{x}^{\bar{\alpha}}(1, t)=R^{\bar{\alpha}}(t) \\
R^{\bar{\alpha}}(t)=\sum_{\beta=1}^{m_{\bar{\alpha}}} T^{\bar{\alpha}_{\circ} \beta} y_{x}^{\bar{\alpha}^{\circ} \beta}(0, t) .
\end{gathered}
$$

According to (12) and (13), the Laplacian transform of (14) yields

$$
\begin{gathered}
f^{\bar{\alpha}}(x, \lambda)=c^{\bar{\alpha}}\left[F^{\bar{\alpha}}(\lambda) \cosh \lambda \rho^{\bar{\alpha}} x-G^{\bar{\alpha}}(\lambda) \sinh \lambda \rho^{\bar{\alpha}} x\right] \\
f^{\bar{\alpha} \circ \beta}(x, \lambda) \\
=c^{\bar{\alpha} \circ \beta}\left[F^{\bar{\alpha}^{\alpha} \beta}(\lambda) \cosh \lambda \rho^{\bar{\alpha} \circ \beta} x-G^{\bar{\alpha} \circ \beta}(\lambda) \sinh \lambda \rho^{\bar{\alpha} \circ \beta} x\right], \\
\quad \beta=1,2, \ldots, m_{\bar{\alpha}} \\
f^{\bar{\alpha}}(1, \lambda)=f^{\bar{\alpha} \circ \beta}(0, \lambda), \quad \beta=1,2, \ldots, m_{\bar{\alpha}} \\
T^{\bar{\alpha}} f_{x}^{\bar{\alpha}}(1, \lambda)=\widehat{R}^{\bar{\alpha}}(\lambda) \\
\widehat{R}^{\bar{\alpha}}(\lambda)=\sum_{\beta=1}^{m_{\bar{\alpha}}} T^{\bar{\alpha} \circ \beta} f_{x}^{\bar{\alpha}^{\alpha} \beta}(0, \lambda) .
\end{gathered}
$$

The following theorem indicates the recursive relations between the coefficients $F^{\bar{\alpha}}(\lambda), G^{\bar{\alpha}}(\lambda)$, and $F^{\bar{\alpha} \beta}(\lambda), G^{\bar{\alpha} \circ \beta}(\lambda)$, $\beta=1,2, \ldots, m_{\bar{\alpha}}$ of the adjacent edges. It provides us with the easy manner for the exponential stability analysis.

Theorem 1. Let $G$ be a complex wave network and $\mathscr{A}$ be a subtree of $G$ described by (11). Assume that $\lambda \neq 0$. Then for any $\bar{\alpha} \in \mathscr{J}_{M}$, the recursive expressions of $F^{\bar{\alpha}}(\lambda)$ and $G^{\bar{\alpha}}(\lambda)$ in $F^{\bar{\alpha} \beta}(\lambda), G^{\bar{\alpha} \alpha \beta}(\lambda), \beta=1,2, \ldots, m_{\bar{\alpha}}$ are given by

$$
\begin{aligned}
& F^{\bar{\alpha}}(\lambda)=\frac{1}{T^{\bar{\alpha}} \rho^{\bar{\alpha}}}\left[T^{\bar{\alpha}} \rho^{\bar{\alpha}} \prod_{\beta=1}^{m_{\bar{\alpha}}} F^{\bar{\alpha} \circ \beta}(\lambda) \cosh \lambda \rho^{\bar{\alpha}}\right. \\
& +\sinh \lambda \rho^{\bar{\alpha}} \sum_{\beta=1}^{m_{\bar{\alpha}}} T^{\bar{\alpha} \circ \beta} \rho^{\bar{\alpha} \circ \beta} G^{\bar{\alpha} \circ \beta}(\lambda) \\
& \left.\times \prod_{\gamma=1, \gamma \neq \beta}^{m_{\bar{\alpha}}} F^{\bar{\alpha} \circ \gamma}(\lambda)\right] \\
& G^{\bar{\alpha}}(\lambda)=\frac{1}{T^{\bar{\alpha}} \rho^{\bar{\alpha}}}\left[T^{\bar{\alpha}} \rho^{\bar{\alpha}} \prod_{\beta=1}^{m_{\bar{\alpha}}} F^{\bar{\alpha} \beta}(\lambda) \sinh \lambda \rho^{\bar{\alpha}}\right. \\
& +\cosh \lambda \rho^{\bar{\alpha}} \sum_{\beta=1}^{m_{\bar{\alpha}}} T^{\bar{\alpha} \circ \beta} \rho^{\bar{\alpha} \circ \beta} G^{\bar{\alpha} \circ \beta}(\lambda) \\
& \left.\times \prod_{\gamma=1, \gamma \neq \beta}^{m_{\bar{\alpha}}} F^{\bar{\alpha} \gamma}(\lambda)\right] .
\end{aligned}
$$

Moreover, if

$$
\inf _{\sigma \in \mathbb{R}}\left|F^{\bar{\alpha} \circ \beta}(i \sigma)\right|>0, \quad \Re\left(G^{\bar{\alpha} \circ \beta}(i \sigma) \overline{F^{\bar{\alpha} \circ \beta}(i \sigma)}\right)>0
$$

hold for every $\beta=1,2, \ldots, m_{\bar{\alpha}}$, then

$$
\inf _{\sigma \in \mathbb{R}}\left|F^{\bar{\alpha}}(i \sigma)\right|>0, \quad \mathfrak{R}\left(G^{\bar{\alpha}}(i \sigma) \overline{F^{\bar{\alpha}}(i \sigma)}\right)>0 .
$$

Proof. According to (15), we obtain that

$$
\begin{aligned}
& f^{\bar{\alpha}}(1, \lambda)=c^{\bar{\alpha}}\left[F^{\bar{\alpha}}(\lambda) \cosh \lambda \rho^{\bar{\alpha}}-G^{\bar{\alpha}}(\lambda) \sinh \lambda \rho^{\bar{\alpha}}\right] \\
& \widehat{R}^{\bar{\alpha}}(\lambda)=T^{\bar{\alpha}} f_{x}^{\bar{\alpha}}(1, \lambda) \\
& =\lambda c^{\bar{\alpha}} T^{\bar{\alpha}} \rho^{\bar{\alpha}}\left[F^{\bar{\alpha}}(\lambda) \sinh \lambda \rho^{\bar{\alpha}}-G^{\bar{\alpha}}(\lambda) \cosh \lambda \rho^{\bar{\alpha}}\right],
\end{aligned}
$$

which indicates

$F^{\bar{\alpha}}(\lambda)$

$$
=\frac{1}{c^{\bar{\alpha}} T^{\bar{\alpha}} \rho^{\bar{\alpha}}}\left[T^{\bar{\alpha}} \rho^{\bar{\alpha}} f^{\bar{\alpha}}(1, \lambda) \cosh \lambda \rho^{\bar{\alpha}}-\frac{1}{\lambda} \widehat{R}^{\bar{\alpha}}(\lambda) \sinh \lambda \rho^{\bar{\alpha}}\right]
$$

$G^{\bar{\alpha}}(\lambda)$

$$
=\frac{1}{c^{\bar{\alpha}} T^{\bar{\alpha}} \rho^{\bar{\alpha}}}\left[T^{\bar{\alpha}} \rho^{\bar{\alpha}} f^{\bar{\alpha}}(1, \lambda) \sinh \lambda \rho^{\bar{\alpha}}-\frac{1}{\lambda} \widehat{R}^{\bar{\alpha}}(\lambda) \cosh \lambda \rho^{\bar{\alpha}}\right] .
$$

Note that

$$
\begin{aligned}
& f^{\bar{\alpha}}(1, \lambda)=f^{\bar{\alpha} \beta \beta}(0, \lambda)=c^{\bar{\alpha} \beta \beta} F^{\bar{\alpha} \propto \beta}(\lambda), \quad \beta=1,2, \ldots, m_{\bar{\alpha}}, \\
& \widehat{R}^{\bar{\alpha}}(\lambda)=\sum_{\beta=1}^{m_{\bar{\alpha}}} T^{\bar{\alpha} \circ \beta} f_{x}^{\bar{\alpha} \circ \beta}(0, \lambda)=-\lambda \sum_{\beta=1}^{m_{\bar{\alpha}}} T^{\bar{\alpha} \circ \beta} \rho^{\bar{\alpha} \circ \beta} c^{\bar{\alpha}^{\circ} \beta} G^{\bar{\alpha} \circ \beta}(\lambda) .
\end{aligned}
$$


Substituting the above into (20), we have

$$
\begin{aligned}
& F^{\bar{\alpha}}(\lambda)=\frac{1}{c^{\bar{\alpha}} T^{\bar{\alpha}} \rho^{\bar{\alpha}}}\left[T^{\bar{\alpha}} \rho^{\bar{\alpha}} c^{\bar{\alpha} \propto \beta} F^{\bar{\alpha} \circ \beta}(\lambda) \cosh \lambda \rho^{\bar{\alpha}}\right. \\
& \left.+\sinh \lambda \rho^{\bar{\alpha}} \sum_{\beta=1}^{m_{\bar{\alpha}}} T^{\bar{\alpha} \circ \beta} \rho^{\bar{\alpha} \circ \beta} c^{\bar{\alpha} \circ \beta} G^{\bar{\alpha} \circ \beta}(\lambda)\right] \\
& G^{\bar{\alpha}}(\lambda)=\frac{1}{c^{\bar{\alpha}} T^{\bar{\alpha}} \rho^{\bar{\alpha}}}\left[T^{\bar{\alpha}} \rho^{\bar{\alpha}} c^{\bar{\alpha} \circ \beta} F^{\bar{\alpha} \circ \beta}(\lambda) \sinh \lambda \rho^{\bar{\alpha}}\right. \\
& \left.+\cosh \lambda \rho^{\bar{\alpha}} \sum_{\beta=1}^{m_{\bar{\alpha}}} T^{\bar{\alpha} \circ \beta} \rho^{\bar{\alpha} \circ \beta} c^{\bar{\alpha}^{\circ} \beta} G^{\bar{\alpha} \circ \beta}(\lambda)\right] .
\end{aligned}
$$

Take

$$
c^{\bar{\alpha} \circ \beta}=c^{\bar{\alpha}} \prod_{\gamma=1, \gamma \neq \beta}^{m_{\bar{\alpha}}} F^{\bar{\alpha} \circ \gamma}(\lambda), \quad \beta=1,2, \ldots, m_{\bar{\alpha}},
$$

then (22) yields

$$
\begin{aligned}
& F^{\bar{\alpha}}(\lambda)=\frac{1}{T^{\bar{\alpha}} \rho^{\bar{\alpha}}}\left[T^{\bar{\alpha}} \rho^{\bar{\alpha}} \prod_{\beta=1}^{m_{\bar{\alpha}}} F^{\bar{\alpha} \beta}(\lambda) \cosh \lambda \rho^{\bar{\alpha}}\right. \\
& +\sinh \lambda \rho^{\bar{\alpha}} \sum_{\beta=1}^{m_{\bar{\alpha}}} T^{\bar{\alpha} \diamond \beta} \rho^{\bar{\alpha} \beta \beta} G^{\bar{\alpha} ॰ \beta}(\lambda) \\
& \left.\times \prod_{\gamma=1, \gamma \neq \beta}^{m_{\bar{\alpha}}} F^{\bar{\alpha} \gamma}(\lambda)\right] \\
& G^{\bar{\alpha}}(\lambda)=\frac{1}{T^{\bar{\alpha}} \rho^{\bar{\alpha}}}\left[T^{\bar{\alpha}} \rho^{\bar{\alpha}} \prod_{\beta=1}^{m_{\bar{\alpha}}} F^{\bar{\alpha} \circ \beta}(\lambda) \sinh \lambda \rho^{\bar{\alpha}}\right. \\
& +\cosh \lambda \rho^{\bar{\alpha}} \sum_{\beta=1}^{m_{\bar{\alpha}}} T^{\bar{\alpha} \circ \beta} \rho^{\bar{\alpha} \circ \beta} G^{\bar{\alpha} \circ \beta}(\lambda) \\
& \left.\times \prod_{\gamma=1, \gamma \neq \beta}^{m_{\bar{\alpha}}} F^{\bar{\alpha} \circ \gamma}(\lambda)\right]
\end{aligned}
$$

Thus we have proved (16) in the theorem.

Since $\inf _{\sigma \in \mathbb{R}}\left|F^{\bar{\alpha} \circ \beta}(i \sigma)\right|>0, \beta=1,2, \ldots, m_{\bar{\alpha}}$, we derive from (16) that

$$
\begin{aligned}
& \frac{T^{\bar{\alpha}} \rho^{\bar{\alpha}} F^{\bar{\alpha}}(i \sigma)}{\prod_{\beta=1}^{m_{\bar{\alpha}}} F^{\bar{\alpha} \circ \beta}(i \sigma)} \\
& \quad=T^{\bar{\alpha}} \rho^{\bar{\alpha}} \cos \sigma \rho^{\bar{\alpha}} \\
& \quad+i \sin \sigma \rho^{\bar{\alpha}} \sum_{\beta=1}^{m_{\bar{\alpha}}} T^{\bar{\alpha} \circ \beta} \rho^{\bar{\alpha} \circ \beta} \frac{G^{\bar{\alpha} \circ \beta}(i \sigma)}{F^{\bar{\alpha} \circ \beta}(i \sigma)}
\end{aligned}
$$

$$
\begin{aligned}
= & T^{\bar{\alpha}} \rho^{\bar{\alpha}} \cos \sigma \rho^{\bar{\alpha}} \\
& -\sin \sigma \rho^{\bar{\alpha}} \sum_{\beta=1}^{m_{\bar{\alpha}}} T^{\bar{\alpha} \circ \beta} \rho^{\bar{\alpha} \circ \beta} \frac{\mathfrak{J}\left(G^{\bar{\alpha} \circ \beta}(i \sigma) \overline{F^{\bar{\alpha} \circ \beta}(i \sigma)}\right)}{\left|F^{\bar{\alpha} \circ \beta}(i \sigma)\right|^{2}} \\
& +i \sin \sigma \rho^{\bar{\alpha}} \sum_{\beta=1}^{m_{\bar{\alpha}}} T^{\bar{\alpha} \circ \beta} \rho^{\bar{\alpha} \circ \beta} \frac{\Re\left(G^{\bar{\alpha} \circ \beta}(i \sigma) \overline{F^{\bar{\alpha} \circ \beta}(i \sigma)}\right)}{\left|F^{\bar{\alpha} \circ \beta}(i \sigma)\right|^{2}} .
\end{aligned}
$$

Thus the assumption (17) reads that

$$
\inf _{\sigma \in \mathbb{R}}\left|F^{\bar{\alpha}}(i \sigma)\right|>0 \text {. }
$$

To complete the proof, we deduce from (16) that

$$
\begin{aligned}
& \left(T^{\bar{\alpha}} \rho^{\bar{\alpha}}\right)^{2} G^{\bar{\alpha}}(i \sigma) \overline{F^{\bar{\alpha}}(i \sigma)} \\
& \quad=\left[i T^{\bar{\alpha}} \rho^{\bar{\alpha}} \prod_{\beta=1}^{m_{\bar{\alpha}}} F^{\bar{\alpha} \circ \beta}(i \sigma) \sin \sigma \rho^{\bar{\alpha}}\right.
\end{aligned}
$$

$$
\begin{aligned}
& \left.+\cos \lambda \rho^{\bar{\alpha}} \sum_{\beta=1}^{m_{\bar{\alpha}}} T^{\bar{\alpha} \circ \beta} \rho^{\bar{\alpha} \circ \beta} G^{\bar{\alpha} \circ \beta}(i \sigma) \prod_{\gamma=1, \gamma \neq \beta}^{m_{\bar{\alpha}}} F^{\bar{\alpha} \circ \gamma}(i \sigma)\right] \\
& {\left[T^{\bar{\alpha}} \rho^{\bar{\alpha}} \prod_{\beta=1}^{m_{\bar{\alpha}}} \overline{F^{\bar{\alpha} \circ \beta}(i \sigma)} \cos \sigma \rho^{\bar{\alpha}}\right.}
\end{aligned}
$$$$
\left.-i \sin \sigma \rho^{\bar{\alpha}} \sum_{\beta=1}^{m_{\bar{\alpha}}} T^{\bar{\alpha} \circ \beta} \rho^{\bar{\alpha} \circ \beta} \overline{G^{\bar{\alpha} \circ \beta}(i \sigma)} \prod_{\gamma=1, \gamma \neq \beta}^{m_{\bar{\alpha}}} \overline{F^{\bar{\alpha} \circ}(i \sigma)}\right]
$$$$
=\frac{i}{2}\left(T^{\bar{\alpha}} \rho^{\bar{\alpha}}\right)^{2} \prod_{\beta=1}^{m_{\bar{\alpha}}}\left|F^{\bar{\alpha} \beta}(i \sigma)\right|^{2} \sin 2 \sigma \rho^{\bar{\alpha}}
$$$$
-\frac{i}{2} \sin 2 \sigma \rho^{\bar{\alpha}} \sum_{\beta=1}^{m_{\bar{\alpha}}}\left|T^{\bar{\alpha} \circ \beta} \rho^{\bar{\alpha} \circ \beta} G^{\bar{\alpha} \circ \beta}(i \sigma) \prod_{\gamma=1, \gamma \neq \beta}^{m_{\bar{\alpha}}} F^{\bar{\alpha} \circ \gamma}(i \sigma)\right|^{2}
$$$$
+T^{\bar{\alpha}} \rho^{\bar{\alpha}} \sin ^{2} \sigma \rho^{\bar{\alpha}} \prod_{\beta=1}^{m_{\bar{\alpha}}} F^{\bar{\alpha} \circ \beta}(i \sigma)
$$$$
\times \sum_{\beta=1}^{m_{\bar{\alpha}}} T^{\bar{\alpha} \circ \beta} \rho^{\bar{\alpha} \circ \beta} \overline{G^{\bar{\alpha} \circ \beta}(i \sigma)} \prod_{\gamma=1, \gamma \neq \beta}^{m_{\bar{\alpha}}} \overline{F^{\bar{\alpha} \circ \gamma}(i \sigma)}
$$$$
+T^{\bar{\alpha}} \rho^{\bar{\alpha}} \cos ^{2} \sigma \rho \rho_{\beta=1}^{\bar{\alpha}} \prod_{\bar{\alpha}}^{\overline{F^{\bar{\alpha}} \beta}(i \sigma)}
$$$$
\times \sum_{\beta=1}^{m_{\bar{\alpha}}} T^{\bar{\alpha} \circ \beta} \rho^{\bar{\alpha} \circ \beta} G^{\bar{\alpha} \circ \beta}(i \sigma) \prod_{\gamma=1, \gamma \neq \beta}^{m_{\bar{\alpha}}} F^{\bar{\alpha} \circ \gamma}(i \sigma)
$$$$
:=\Theta_{1}+\Theta_{2}+\Theta_{3}+\Theta_{4} \text {, }
$$ 
where $\mathfrak{R} \Theta_{1}=\mathfrak{R} \Theta_{2}=0$, and

$$
\begin{aligned}
\Re\left(\Theta_{3}+\Theta_{4}\right)= & T^{\bar{\alpha}} \rho^{\bar{\alpha}} \sum_{\beta=1}^{m_{\bar{\alpha}}} T^{\bar{\alpha} \circ \beta} \rho^{\bar{\alpha} \circ \beta} \\
& \times \Re\left(\overline{G^{\bar{\alpha} \alpha \beta}(i \sigma)} F^{\bar{\alpha} \circ \beta}(i \sigma) \sin ^{2} \sigma \rho^{\bar{\alpha}}\right. \\
& \left.+G^{\bar{\alpha} \circ \beta}(i \sigma) \overline{F^{\bar{\alpha}} \beta(i \sigma)} \cos ^{2} \sigma \rho^{\bar{\alpha}}\right) \\
& \times \prod_{\gamma=1, \gamma \neq \beta}^{m_{\bar{\alpha}}}\left|F^{\bar{\alpha} \circ \gamma}(i \sigma)\right|^{2} \\
= & T^{\bar{\alpha}} \rho^{\bar{\alpha}} \sum_{\beta=1}^{m_{\bar{\alpha}}} T^{\bar{\alpha} \circ \beta} \rho^{\bar{\alpha} \alpha \beta} \Re\left(G^{\bar{\alpha} \alpha \beta}(i \sigma) \overline{F^{\bar{\alpha} \circ \beta}(i \sigma)}\right) \\
& \times \prod_{\gamma=1, \gamma \neq \beta}^{m_{\bar{\alpha}}}\left|F^{\bar{\alpha} \circ \gamma}(i \sigma)\right|^{2} .
\end{aligned}
$$

Thus

$$
\begin{aligned}
& \Re\left(G^{\bar{\alpha}}(i \sigma) \overline{F^{\bar{\alpha}}(i \sigma)}\right) \\
& =\frac{1}{T^{\bar{\alpha}} \rho^{\bar{\alpha}}} \sum_{\beta=1}^{m_{\bar{\alpha}}} T^{\bar{\alpha} \circ \beta} \rho^{\bar{\alpha} \circ \beta} \\
& \quad \times \Re\left(G^{\bar{\alpha} \circ \beta}(i \sigma) \overline{F^{\bar{\alpha} \circ \beta}(i \sigma)}\right) \prod_{\gamma=1, \gamma \neq \beta}^{m_{\bar{\alpha}}}\left|F^{\bar{\alpha} \circ \gamma}(i \sigma)\right|^{2} .
\end{aligned}
$$

Therefore the assumption (17) together with the equality above implies that

$$
\mathfrak{R}\left(G^{\bar{\alpha}}(i \sigma) \overline{F^{\bar{\alpha}}(i \sigma)}\right)>0 .
$$

The proof is then complete.

Remark 2. In (11), there is no interior controller. If a velocity feedback controller is equipped at some interior vertex $v^{\bar{\alpha}}$, $\bar{\alpha} \in \mathscr{J}_{M}$, then we only have to add a term $-a^{\bar{\alpha}} y_{t}^{\bar{\alpha}}(1, t)$ to $R^{\bar{\alpha}}(t)$, where $a^{\bar{\alpha}}$ is the nonnegative feedback gain constant, and $-a^{\bar{\alpha}} y_{t}^{\bar{\alpha}}(1, t)$ can be regarded as a branching damping. In this situation, the results in Theorem 1 can be proved similarly. The only difference is that the recursive expression (16) is modified by

$$
\begin{aligned}
& F^{\bar{\alpha}}(\lambda)=\frac{1}{T^{\bar{\alpha}} \rho^{\bar{\alpha}}}\left[w^{\bar{\alpha}}(\lambda) \prod_{\beta=1}^{m_{\bar{\alpha}}} F^{\bar{\alpha} \circ \beta}(\lambda)\right. \\
&+\sinh \lambda \rho^{\bar{\alpha}} \sum_{\beta=1}^{m_{\bar{\alpha}}} T^{\bar{\alpha} \circ \beta} \rho^{\bar{\alpha} \circ \beta} G^{\bar{\alpha} \circ \beta}(\lambda) \\
&\left.\times \prod_{\gamma=1, \gamma \neq \beta}^{m_{\bar{\alpha}}} F^{\bar{\alpha} \circ \gamma}(\lambda)\right]
\end{aligned}
$$

$$
\begin{aligned}
& G^{\bar{\alpha}}(\lambda)=\frac{1}{T^{\bar{\alpha}} \rho^{\bar{\alpha}}}\left[v^{\bar{\alpha}}(\lambda) \prod_{\beta=1}^{m_{\bar{\alpha}}} F^{\bar{\alpha} \circ \beta}(\lambda)\right. \\
&+\cosh \lambda \rho^{\bar{\alpha}} \sum_{\beta=1}^{m_{\bar{\alpha}}} T^{\bar{\alpha} \circ \beta} \rho^{\bar{\alpha} \circ \beta} G^{\bar{\alpha} \alpha \beta}(\lambda) \\
&\left.\times \prod_{\gamma=1, \gamma \neq \beta}^{m_{\bar{\alpha}}} F^{\bar{\alpha} \alpha \gamma}(\lambda)\right],
\end{aligned}
$$

where

$$
\begin{array}{r}
w^{\bar{\alpha}}(\lambda)=T^{\bar{\alpha}} \rho^{\bar{\alpha}} \cosh \lambda \rho^{\bar{\alpha}}+a^{\bar{\alpha}} \sinh \lambda \rho^{\bar{\alpha}} \\
v^{\bar{\alpha}}(\lambda)=T^{\bar{\alpha}} \rho^{\bar{\alpha}} \sinh \lambda \rho^{\bar{\alpha}}+a^{\bar{\alpha}} \cosh \lambda \rho^{\bar{\alpha}}, \\
a^{\bar{\alpha}} \geq 0 .
\end{array}
$$

Now we consider the special case that $\mathscr{A}$ is a tree-shaped network. We apply Theorem 1 to its stability analysis. Herein, the boundary conditions are set to be

$$
T^{\bar{\alpha}} y_{x}^{\bar{\alpha}}(1, t)=R^{\bar{\alpha}}(t), \quad \bar{\alpha} \in \mathscr{F}_{S} .
$$

If the root $\mathscr{R}$ is clamped, that is, $y(0, t)=0$, then we get from its Laplacian transform $f(0, \lambda)=0$ that $F(\lambda)=0$, which is the characteristic equation of $\mathscr{A}$. Using (16) in Theorem 1 , we obtain the recursive expression of $F(\lambda)$. Moreover, if we choose appropriate $R^{\bar{\alpha}}(t), \bar{\alpha} \in \mathscr{J}_{S}$ such that

$$
\inf _{\sigma \in \mathbb{R}}\left|F^{\bar{\alpha}}(i \sigma)\right|>0, \quad \Re\left(G^{\bar{\alpha}}(i \sigma) \overline{F^{\bar{\alpha}}(i \sigma)}\right)>0, \quad \forall \bar{\alpha} \in \mathscr{F}_{S},
$$

then the second assertion of Theorem 1 says that

$$
\inf _{\sigma \in \mathbb{R}}|F(i \sigma)|>0
$$

The inequality (35) guarantees the exponential stability of $\mathscr{A}$, provided that $\mathscr{A}$ satisfies SDGA. If (34)-(35) are not true, the exponential stability of $\mathscr{A}$ no longer holds. However, we can still obtain the asymptotic stability of it as long as $F(i \sigma) \neq 0$, $\sigma \in \mathbb{R}$.

If the root $\mathscr{R}$ is free, we can similarly analyze the stability. In fact, we can prove similarly that if

$$
\inf _{\sigma \in \mathbb{R}}\left|G^{\bar{\alpha} \circ \beta}(i \sigma)\right|>0, \quad \Re\left(G^{\bar{\alpha} \circ \beta}(i \sigma) \overline{F^{\bar{\alpha} \propto \beta}(i \sigma)}\right)>0
$$

hold for every $\beta=1,2, \ldots, m_{\bar{\alpha}}$, then

$$
\inf _{\sigma \in \mathbb{R}}\left|G^{\bar{\alpha}}(i \sigma)\right|>0, \quad \mathfrak{R}\left(G^{\bar{\alpha}}(i \sigma) \overline{F^{\bar{\alpha}}(i \sigma)}\right)>0 .
$$

In the same way, Theorem 1 can also be applied for the stability analysis of subtrees of complex networks. 


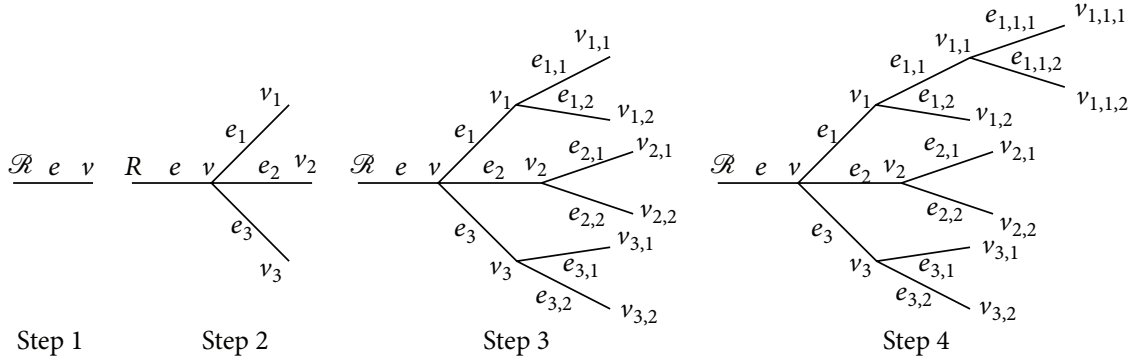

FIGURE 2: The "branching" process of the 12-edge tree-shaped wave network.

\section{Application Example}

In this section, we will give an example of 12-edge tree-shaped wave network as an application to Theorem 1 . We will see that the stability analysis of the controlled system can be simply carried out by our approach.

The structure of the network is shown in the rightmost of Figure 2. It is viewed as branching out from a single edge; the process is divided into 4 steps (also see Figure 2).

The network is described by

$$
\begin{aligned}
& T^{\bar{\alpha}} y_{x x}^{\bar{\alpha}}(x, t)=m^{\bar{\alpha}} y_{t t}^{\bar{\alpha}}(x, t), \quad x \in(0,1), t>0, \bar{\alpha} \in \mathcal{J} \\
& y(0, t)=0 \\
& y^{\bar{\alpha}}(1, t)=y^{\bar{\alpha} \circ \beta}(0, t), \quad \bar{\alpha} \in \mathscr{J}_{M}, \quad \beta=1,2 \\
& T^{\bar{\alpha}} y_{x}^{\bar{\alpha}}(1, t)=R^{\bar{\alpha}}(t), \quad \bar{\alpha} \in \mathscr{J}_{M} \\
& R^{\bar{\alpha}}(t)=\sum_{\beta=1}^{2} T^{\bar{\alpha} \circ \beta} y_{x}^{\bar{\alpha} \circ \beta}(0, t), \quad \bar{\alpha} \in \mathscr{J}_{M} \\
& T^{\bar{\alpha}} y_{x}^{\bar{\alpha}}(1, t)=R^{\bar{\alpha}}(t), \quad \bar{\alpha} \in \mathscr{J}_{S},
\end{aligned}
$$

where $\mathscr{J}_{M}=$ empty index, (1), (2), (3), (1,1)\}, $\mathscr{J}_{S}=$ $\{(1,2),(2,1),(2,2),(3,1),(3,2),(1,1,1),(1,1,2)\}$, and $\mathscr{J}=$ $\mathscr{J}_{M} \cup \mathscr{J}_{S}$.

Since no edge branches out from $v^{\bar{\alpha}}, \bar{\alpha} \in \mathscr{J}_{S}$, we take the extra forces to be the collocated velocity feedback control:

$$
R^{\bar{\alpha}}(t)=-a^{\bar{\alpha}} y_{t}^{\bar{\alpha}}(1, t), \quad a^{\bar{\alpha}}>0, \bar{\alpha} \in \mathscr{J}_{S} .
$$

Thus the 12-edge tree-shaped wave network is described by (38)-(39). A standard regulation of (38)-(39) results in its vector-valued form, which falls into the model discussed in [14]. Thus all the theoretic results in [14] hold, among which we emphasize that

(1) the system is well-posed;

(2) $\lambda \neq 0$; and

(3) if $a^{\bar{\alpha}} \neq \sqrt{T^{\bar{\alpha}} / m^{\bar{\alpha}}}, \bar{\alpha} \in \mathscr{J}_{S}$, then the SDGA satisfies.

Moreover, we have the following assertion.
Proposition 3. Let $D(\lambda), \lambda \in \mathbb{C}$ be the characteristic equation of (38)-(39). Suppose that $a^{\bar{\alpha}} \neq \sqrt{T^{\bar{\alpha}} / m^{\bar{\alpha}}}, \bar{\alpha} \in \mathcal{J}_{S}$. If $\inf _{\sigma \in \mathbb{R}}|D(i \sigma)| \neq 0$, then the system (38)-(39) is exponentially stable.

In the sequel, we will deduce the characteristic equation by our approach shown in Section 2, prove inf $\operatorname{in\mathbb {R}}_{\sigma}|D(i \sigma)| \neq 0$ by Theorem 1 , and obtain the exponential stability of system (38)-(39) according to Proposition 3.

To this end, we first derive the recursive expressions of the coefficients $F^{\bar{\alpha}}(\lambda)$ and $G^{\bar{\alpha}}(\lambda), \bar{\alpha} \in \mathcal{J}$ in its branching order. Taking the Laplacian transform of (38)-(39), one has

$$
\begin{gathered}
f^{\bar{\alpha}}(x, \lambda)=c^{\bar{\alpha}}\left[F^{\bar{\alpha}}(\lambda) \cosh \lambda \rho^{\bar{\alpha}} x-G^{\bar{\alpha}}(\lambda) \sinh \lambda \rho^{\bar{\alpha}} x\right] \\
\rho^{\bar{\alpha}}=\sqrt{\frac{m^{\bar{\alpha}}}{T^{\bar{\alpha}}}, \quad \bar{\alpha} \in \mathcal{J}} \\
f(0, \lambda)=0, \\
f(1, \lambda)=f^{(k)}(0, \lambda) \\
T f_{x}(1, \lambda)=\widehat{R}(\lambda)=\sum_{k=1}^{3} T^{(k)} f_{x}^{(k)}(0, \lambda) \\
f^{(k)}(1, \lambda)=f^{(k, j)}(0, \lambda) \\
T^{(k)} f_{x}^{(k)}(1, \lambda)=\widehat{R}^{(k)}(\lambda)=\sum_{j=1}^{2} T^{(k, j)} f_{x}^{(k, j)}(0, \lambda) \\
T^{(1,1)}(1, \lambda)=f^{(1,1, l)}(0, \lambda) \\
f_{x}^{\bar{\alpha}}(1, \lambda)=\widehat{R}^{\bar{\alpha}}(\lambda)=-\lambda a^{\bar{\alpha}} f^{\bar{\alpha}}(1, \lambda), \quad \bar{\alpha} \in \mathscr{J}_{S} . \\
T^{(1,1)} f_{x}^{(1,1)}(1, \lambda)=\widehat{R}^{(1,1)}(\lambda)=\sum_{l=1}^{2} T^{(1,1, l)} f_{x}^{(1,1, l)}(0, \lambda)
\end{gathered}
$$

Firstly, substituting (40) into (41) indicates

$$
F(\lambda)=0
$$

which is the characteristic equation of the system (38)-(39) to be determined. 

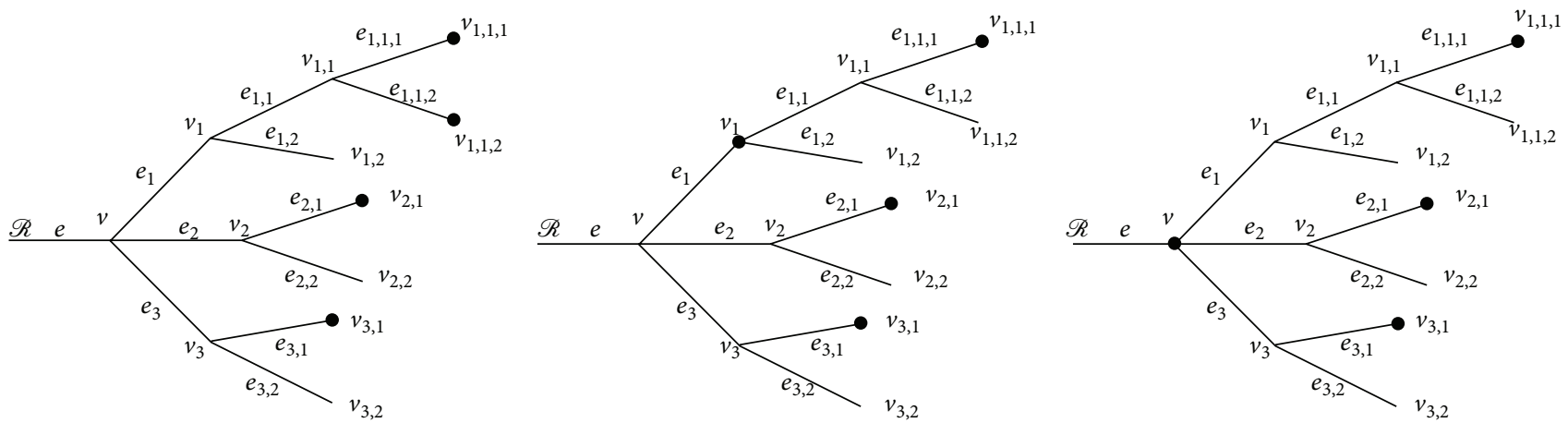

FIGURE 3: Fewer controllers are needed for the exponential stability.

Then, according to (16) in Theorem 1, we get the recursive expressions of $F^{\bar{\alpha}}(\lambda)$ and $G^{\bar{\alpha}}(\lambda), \bar{\alpha} \in \mathscr{F}_{M}$ :

$$
\begin{aligned}
& F(\lambda)=\frac{1}{T \rho}\left[T \rho \cosh \lambda \rho \prod_{k=1}^{3} F^{(k)}(\lambda)\right. \\
& +\sinh \lambda \rho \sum_{k=1}^{3} T^{(k)} \rho^{(k)} G^{(k)}(\lambda) \\
& \left.\times \prod_{j=1, j \neq k}^{3} F^{(j)}(\lambda)\right] \\
& G(\lambda)=\frac{1}{T \rho}\left[T \rho \sinh \lambda \rho \prod_{k=1}^{3} F^{(k)}(\lambda)\right. \\
& +\cosh \lambda \rho \sum_{k=1}^{3} T^{(k)} \rho^{(k)} G^{(k)}(\lambda) \\
& \left.\times \prod_{j=1, j \neq k}^{3} F^{(j)}(\lambda)\right] \\
& F^{(k)}(\lambda)=\frac{1}{T^{(k)} \rho^{(k)}} \\
& \times\left[T^{(k)} \rho^{(k)} \cosh \lambda \rho^{(k)} \prod_{j=1}^{2} F^{(k, j)}(\lambda)\right. \\
& +\sinh \lambda \rho^{(k)} \sum_{j=1}^{2} T^{(k, j)} \rho^{(k, j)} G^{(k, j)}(\lambda) \\
& \left.\times \prod_{l=1, l \neq j}^{2} F^{(k, l)}(\lambda)\right] \\
& G^{(k)}(\lambda)=\frac{1}{T^{(k)} \rho^{(k)}}
\end{aligned}
$$

$$
\begin{aligned}
F^{(1,1)}(\lambda)= & \frac{1}{T^{(1,1)} \rho^{(1,1)}} \\
\times & {\left[T^{(1,1)} \rho^{(1,1)} \cosh \lambda \rho^{(1,1)} \prod_{k=1}^{2} F^{(1,1, k)}(\lambda)\right.} \\
& +\sinh \lambda \rho^{(1,1)} \sum_{k=1}^{2} T^{(1,1, k)} \rho^{(1,1, k)} G^{(1,1, k)}(\lambda) \\
G^{(1,1)}(\lambda)= & \left.\quad \times \prod_{j=1, j \neq k}^{2} F^{(1,1, j)}(\lambda)\right] \\
\times & {\left[\begin{array}{l}
1 \\
T^{(1,1)} \rho^{(1,1)}
\end{array}\right] } \\
& +\cosh \lambda \rho^{(1,1)} \sinh ^{(1,1)} \sum_{k=1}^{2} T^{(1,1,1)} \prod_{k=1}^{2} F^{(1,1, k)}(\lambda) \\
& \left.\times \prod_{j=1, j \neq k}^{2} F^{(1,1, k)} G^{(1,1,1, j)}(\lambda)\right] .
\end{aligned}
$$


Finally, we substitute (40) into (43) to get

$$
\begin{array}{r}
F^{\bar{\alpha}}(\lambda)\left(T^{\bar{\alpha}} \rho^{\bar{\alpha}} \sinh \lambda \rho^{\bar{\alpha}}+\alpha^{\bar{\alpha}} \cosh \lambda \rho^{\bar{\alpha}}\right) \\
-G^{\bar{\alpha}}(\lambda)\left(T^{\bar{\alpha}} \rho^{\bar{\alpha}} \cosh \lambda \rho^{\bar{\alpha}}+\alpha^{\bar{\alpha}} \sinh \lambda \rho^{\bar{\alpha}}\right)=0, \\
\bar{\alpha} \in \mathscr{J}_{S} .
\end{array}
$$

Thus we can take

$$
\begin{array}{r}
F^{\bar{\alpha}}(\lambda)=T^{\bar{\alpha}} \rho^{\bar{\alpha}} \cosh \lambda \rho^{\bar{\alpha}}+a^{\bar{\alpha}} \sinh \lambda \rho^{\bar{\alpha}} \\
G^{\bar{\alpha}}(\lambda)=T^{\bar{\alpha}} \rho^{\bar{\alpha}} \sinh \lambda \rho^{\bar{\alpha}}+a^{\bar{\alpha}} \cosh \lambda \rho^{\bar{\alpha}}, \\
\bar{\alpha} \in \mathscr{J}_{s} .
\end{array}
$$

By now, one has obtained all the recursive expressions (44)-(49) of the coefficients $F^{\bar{\alpha}}(\lambda)$ and $G^{\bar{\alpha}}(\lambda), \bar{\alpha} \in \mathscr{J}$, which form the characteristic equation of (38)-(39). Now we will prove the exponential stability of the system (38)-(39). Proposition 3 says it suffices to prove that $F(\lambda)$ defined in (45) satisfies $\inf _{\sigma \in \mathbb{R}}|F(i \sigma)|>0$. We will verify this by easily estimating the infimums of (44)-(49) in the inverse order.

By (49), a direct calculation leads to

$$
\begin{aligned}
& F^{\bar{\alpha}}(i \sigma)=T^{\bar{\alpha}} \rho^{\bar{\alpha}} \cos \sigma \rho^{\bar{\alpha}}+i a^{\bar{\alpha}} \sin \sigma \rho^{\bar{\alpha}}, \\
& G^{\bar{\alpha}}(i \sigma)=i T^{\bar{\alpha}} \rho^{\bar{\alpha}} \sin \sigma \rho^{\bar{\alpha}}+a^{\bar{\alpha}} \cos \sigma \rho^{\bar{\alpha}} .
\end{aligned}
$$

Thus one has

$$
\inf _{\sigma \in \mathbb{R}}\left|F^{\bar{\alpha}}(i \sigma)\right|=\inf _{\sigma \in \mathbb{R}}\left|G^{\bar{\alpha}}(i \sigma)\right|=\min \left\{a^{\bar{\alpha}}, T^{\bar{\alpha}} \rho^{\bar{\alpha}}\right\}>0
$$

for $\bar{\alpha} \in \mathscr{J}_{S}$.

Moreover, it holds that

$$
\begin{aligned}
& \Re\left(G^{\bar{\alpha}}(i \sigma) \overline{F^{\bar{\alpha}}(i \sigma)}\right) \\
& =\Re\left(\left(T^{\bar{\alpha}} \rho^{\bar{\alpha}} \cos \sigma \rho^{\bar{\alpha}}-i a^{\bar{\alpha}} \sin \sigma \rho^{\bar{\alpha}}\right)\right. \\
& \left.\times\left(i T^{\bar{\alpha}} \rho^{\bar{\alpha}} \sin \sigma \rho^{\bar{\alpha}}+a^{\bar{\alpha}} \cos \sigma \rho^{\bar{\alpha}}\right)\right) \\
& =a^{\bar{\alpha}} T^{\bar{\alpha}} \rho^{\bar{\alpha}}>0, \quad \bar{\alpha} \in \mathscr{J}_{S} .
\end{aligned}
$$

Thus Theorem 1, (34)-(35), and (51)-(52) read that

$$
\inf _{\sigma \in \mathbb{R}}|F(\lambda)|>0 \text {. }
$$

Therefore Proposition 3 indicates the theorem below.

Theorem 4. Let the 12-edge tree-shaped wave network be given by (38)-(39). If $a^{\bar{\alpha}} \neq \sqrt{T^{\bar{\alpha}} / m^{\bar{\alpha}}}, \bar{\alpha} \in \mathcal{J}_{S}$, then it is exponentially stable.

Remark 5. Theorem 4 implies that the tree-shaped wave network is exponentially stabilized by boundary collocated velocity feedback control. We can also study the stability of the system under other control laws. As stated in Remark 2, by adding the term $-a^{\bar{\alpha}} y_{t}^{\bar{\alpha}}(1, t)$ to $R^{\bar{\alpha}}(t), \bar{\alpha} \in \mathcal{J}_{M}$, the characteristic equation of the modified controlled system can be obtained similarly and its stability can be analyzed in the same way. For instance, if $T^{\bar{\alpha}}=\rho^{\bar{\alpha}}=1, \bar{\alpha} \in \mathcal{J}$, fewer controllers are needed for the system to be exponentially stable. This is shown in Figure 3, where the dots stand for the controllers. We omit the details.

\section{Conclusion}

In this paper, we introduce a new approach for the stability analysis of wave networks. Viewing the network as branching out from a single edge, we divide this process into several steps. Then we get the recursive expressions of the Laplacian transform of the adjacent edges of the system in its branching order. These recursive expressions together form the characteristic equation of the system. In the stability analysis, we estimate the infimums of these recursive expressions in the inverse order. Then the exponential stability of the system can be finished in an easy manner. This approach is valid for tree-shaped networks and subtrees of complex networks. For the stability analysis of networks with circuits, it needs some improvements, which will be studied in the future.

\section{Conflict of Interests}

The authors declare that they have no conflict of interests regarding the publication of this paper.

\section{Acknowledgments}

This work was supported by the National Natural Science Foundation of China (NSFC-61174080); the National Natural Science Foundation of China (NSFC-11201476); the Natural Science Foundation of Tianjin City (13JCQNJC04400); and the Fundamental Research Funds for the Central Universities (3122013C002).

\section{References}

[1] M. Tucsnak and G. Weiss, Observation and Control for Operator Semigroups, Birkhäuser Advanced Texts, Birkhäuser, Basel, Switzerland, 2009.

[2] R. Dáger and E. Zuazua, Wave Propagation, Observation and Control in Flexible Multi-Structures, vol. 50 of Mathématiques et Applications, Springer, Berlin, Germany, 2006.

[3] J. E. Lagnese, G. Leugering, and E. J. P. G. Schmidt, Modeling, Analysis and Control of Dynamic Elastic Multi-Link Structures, Birkhäuser, Boston, Mass, USA, 1994.

[4] Y. N. Guo, L. L. Zhang, and Y. X. Zhang, "Non-collocated feedback stabilization of a kind of system described by wave equations," WSEAS Transactions on Mathematics, vol. 8, no. 12, pp. 841-851, 2013.

[5] Y. X. Zhang and G. Q. Xu, "Exponential and super stability of a wave network," Acta Applicandae Mathematicae, vol. 124, pp. 19-41, 2013.

[6] E. Zuazua, "Control and stabilization of waves on 1-d networks," in Modelling and Optimisation of Flows on Networks, vol. 2062 of Lecture Notes in Mathematics, pp. 463-493, Springer, New York, NY, USA, 2013. 
[7] B. Z. Guo, C. Z. Xu, and H. Hammouri, "Output feedback stabilization of a one-dimensional wave equation with an arbitrary time delay in boundary observation," ESAIM: Control, Optimisation and Calculus of Variations, vol. 18, no. 1, pp. 22-35, 2012.

[8] Z. J. Han and G. Q. Xu, "Stabilization and SDG condition of serially connected vibrating strings system with discontinuous displacement," Asian Journal of Control, vol. 14, no. 1, pp. 95-108, 2012.

[9] Y. N. Guo, Y. L. Chen, G. Q. Xu, and Y. X. Zhang, "Exponential stabilization of variable coefficient wave equations in a generic tree with small time-delays in the nodal feedbacks," Journal of Mathematical Analysis and Applications, vol. 395, no. 2, pp. 727746, 2012.

[10] Y. L. Chen, Z. J. Han, G. Q. Xu, and D. Y. Liu, "Exponential stability of string system with variable coefficients under noncollocated feedback controls," Asian Journal of Control, vol. 13, no. 1, pp. 148-163, 2011.

[11] Z. J. Han and G. Q. Xu, "Output feedback stabilisation of a tree-shaped network of vibrating strings with non-collocated observation," International Journal of Control, vol. 84, no. 3, pp. 458-475, 2011.

[12] Y. N. Guo and G. Q. Xu, "Stability and Riesz basis property for general network of strings," Journal of Dynamical and Control Systems, vol. 15, no. 2, pp. 223-245, 2009.

[13] J. Valein and E. Zuazua, "Stabilization of the wave equation on 1-D networks," SIAM Journal on Control and Optimization, vol. 48, no. 4, pp. 2771-2797, 2009.

[14] G. Q. Xu, D. Y. Liu, and Y. Q. Liu, "Abstract second order hyperbolic system and applications to controlled network of strings," SIAM Journal on Control and Optimization, vol. 47, no. 4, pp. 1762-1784, 2008.

[15] K. Ammari and M. Jellouli, "Remark on stabilization of treeshaped networks of strings," Applications of Mathematics, vol. 52, no. 4, pp. 327-343, 2007.

[16] R. Dáger, "Observation and control of vibrations in treeshaped networks of strings," SIAM Journal on Control and Optimization, vol. 43, no. 2, pp. 590-623, 2004.

[17] D. L. Russell, "Controllability and stabilizability theory for linear partial differential equations: recent progress and open questions," SIAM Review, vol. 20, no. 4, pp. 639-739, 1978.

[18] L. F. Ho, "Observabilité frontière de l'équation des ondes," Comptes Rendus des Séances de l'Académie des Sciences I: Mathématique, vol. 302, no. 12, pp. 443-446, 1986.

[19] V. Komornik, Exact Controllability and Stabilization: The Multiplier Method, vol. 36 of Research in Applied Mathematics, Masson, Paris, France; John Wiley \& Sons, New York, NY, USA, 1994.

[20] F. L. Huang, "Characteristic conditions for exponential stability of linear dynamical systems in Hilbert spaces," Annals of Differential Equations, vol. 1, no. 1, pp. 43-56, 1985.

[21] M. Najafi, G. R. Sarhangi, and H. Wang, "Stabilizability of coupled wave equations in parallel under various boundary conditions," IEEE Transactions on Automatic Control, vol. 42, no. 9, pp. 1308-1312, 1997. 


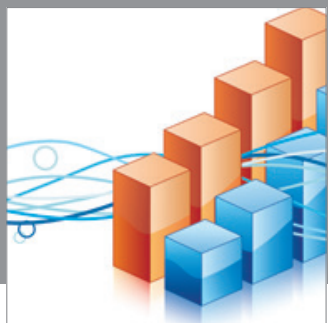

Advances in

Operations Research

mansans

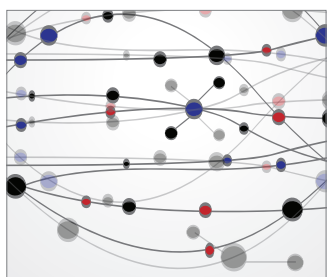

The Scientific World Journal
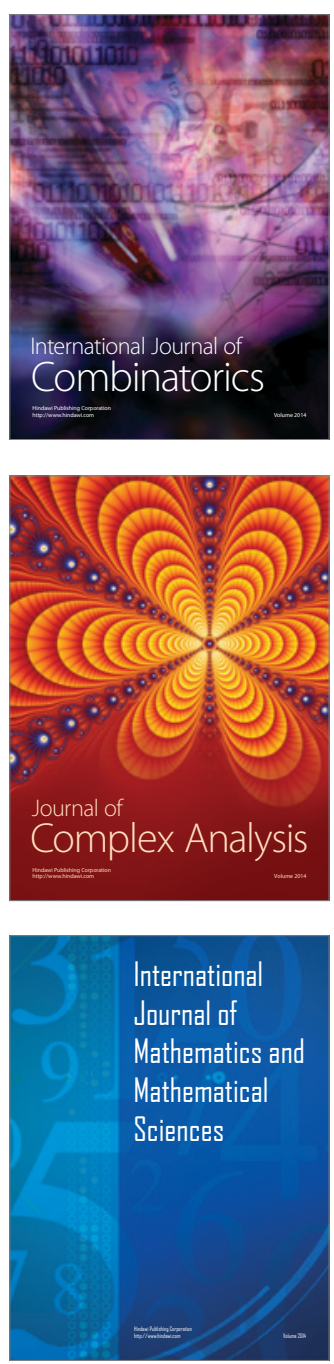
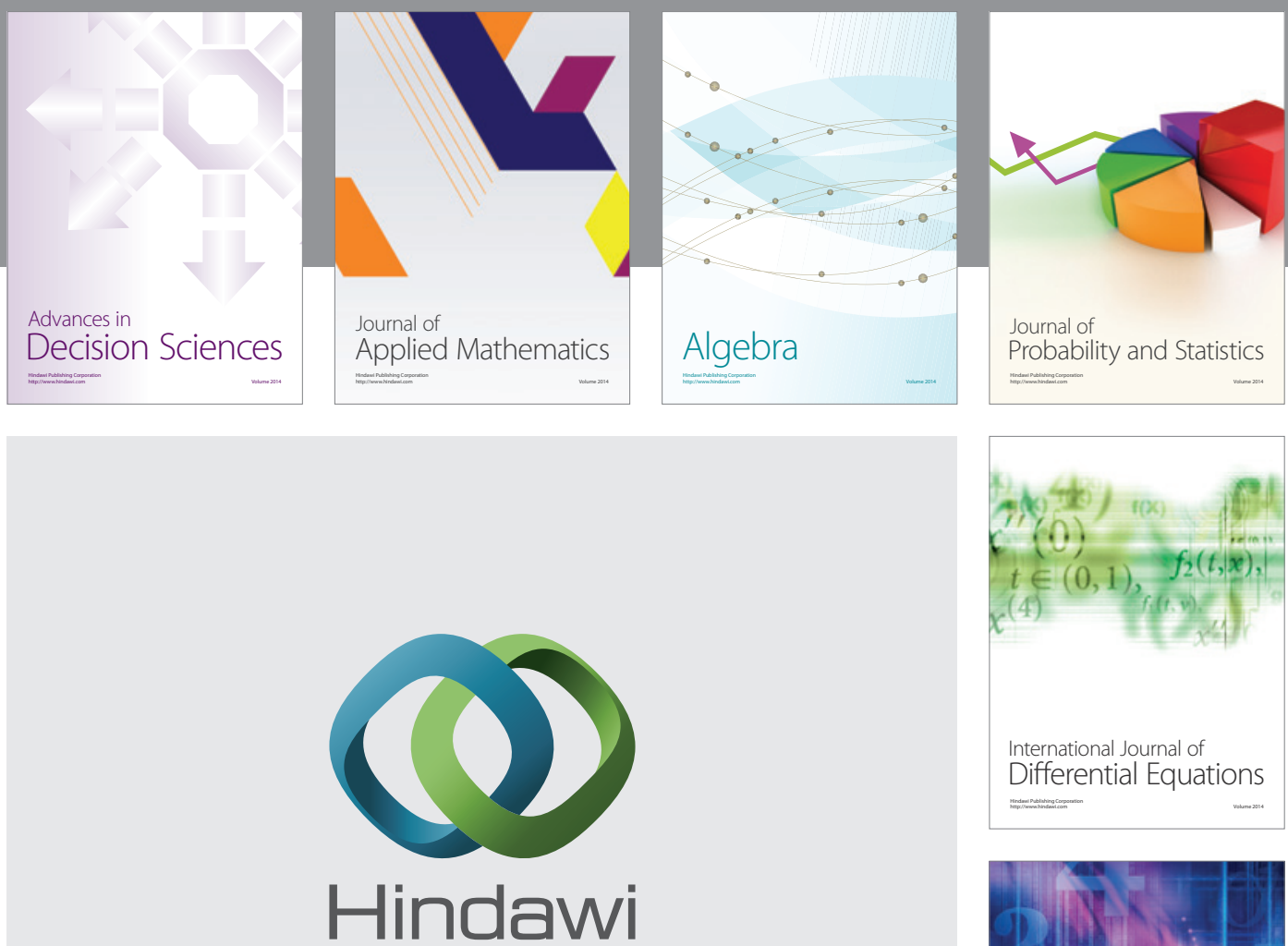

Submit your manuscripts at http://www.hindawi.com
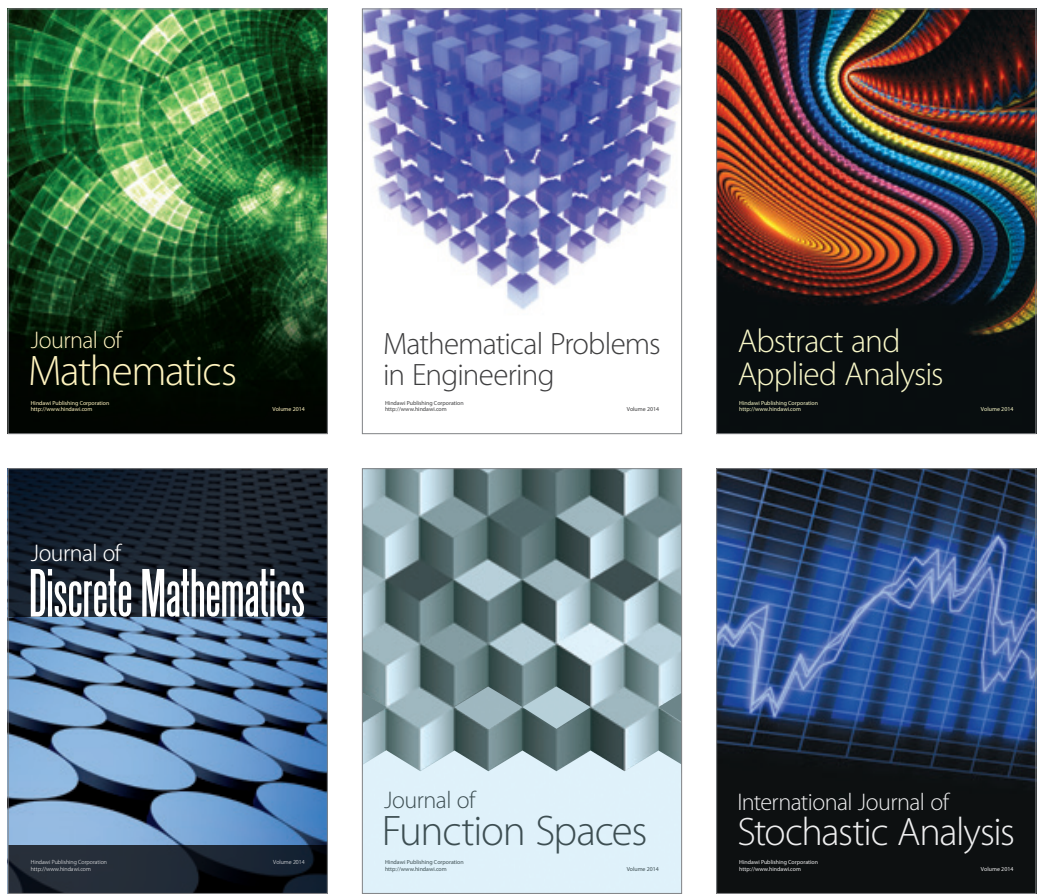

Journal of

Function Spaces

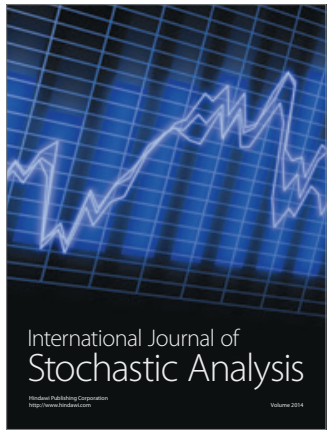

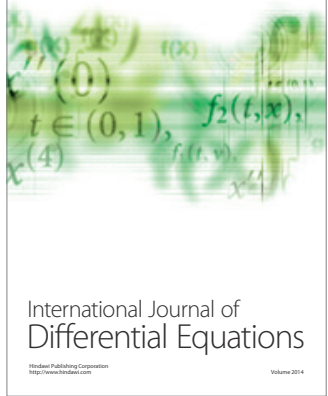
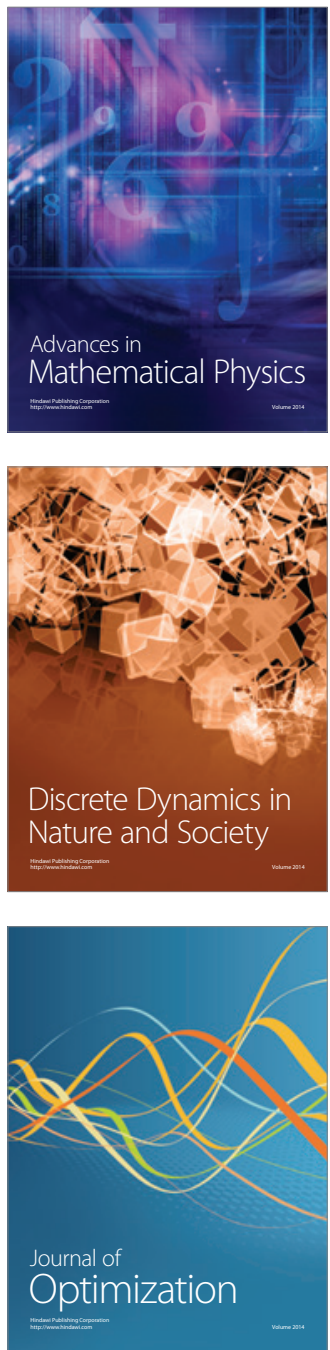